ratio of the slope of the function for negative responses to the slope for positives is close to unity in the case of letters and somewhat greater (about 1.4) for picture stimuli: these slope ratios are essentially the same for both left and right fixation. These data also resemble the previous results in that the serial-position functions (which relate RT for a correct positive response to the position of the test stimulus in the memory set) are increasing; this also occurs for both left and right fixation.

One effect of peripheral presentation appears to be an increase in the slope of the RT functions for letters, relative to the slope for pictures. Previously, it has been found that the slope for pictures is greater than the slope for letters, indicating that the comparison process of Stage 2 is not the same for both stimulus types. In this study, however, the slopes for letters and pictures are nearly equal. Possibly, the use of peripheral vision has the effect of raising only the slope of the function for letter stimuli. Since the theory of memory scanning assumes that the slope represents the time needed for a comparison of the test stimulus with a single memory-set element, the increased slope might indicate that the letter test stimulus representation is less "clear" with peripheral presentation than with foveal and the picture test representation remains the same. Thus, this result appears to agree with the hypothesis that letters are processed in spatial terms, whereas pictures are verbally represented and, therefore, unaffected by visual manipulations.

The present hypothesis was that, in this experiment, letter test stimuli are processed by the right, spatial, hemisphere, whereas pictures are processed by the left, verbal, hemisphere. This leads to the prediction that letters presented to the left hemisphere must cross the corpus callosum before being processed, leading to an increase in the intercept of the corresponding RT function. Similarly, pictures that enter the right hemisphere must be transferred to the left, thus raising the intercept of that function. If it is assumed that the comparison process is unaffected by callosal transmission, the slopes should be the same for right- and left-hemispheric presentation of a stimulus,

An examination of the intercepts of the RT functions for positive responses, negative responses, and both responses combined supports the hypothesis. In each case, as predicted, the intercept of the function for letter stimuli is higher for left-hemispheric presentation than for right, while the reverse holds true for pictures. The difference in intercepts for the combined data of positive and negative responses is $45.0 \mathrm{msec}$ for letters and 33.2 msec for pictures.

Also as predicted. the slope of the function for letter test stimuli presented to the right hemisphere is the same as for presentation to the left. However, this does not hold true for the case of picture stimuli. The slope of the function for picture stimuli presented to the right hemisphere is less than for presentation to the left, indicating a difference in comparison times. One possibility is that this occurs because the transfer of information from the right to the left hemisphere has a facilitative effect on the comparison process. It is also possible that presenting a picture to the left hemisphere, where comparisons are supposed to take place, makes the comparison process more difficult. A third possibility is that some picture stimuli are never transferred at all. This might occur if a picture could be equated with its corresponding letter without the aid of verbalization, enabling the right hemisphere to perform spatial comparisons when it receives a picture stimulus.

This study was intended as a preliminary investigation of the effects of interhemispheric transfer on memory scanning with the letter-and-picture paradigm. Accordingly, a more extensive

experiment is in progress, and it is hoped that that research will support and clarify the present results.

$$
\text { REFERENCES }
$$

BRADSHAW, J. L.. \& PERRIMENT, A. D. Latcrality effects and choice reaction time in a unimanual two-finger task. Perception \& Psychophysics, 1970, 7, 185-188.

FILBEY, R. A., \& GAZZANIGA, M. S. Splitting the normal brain with reaction time Psychonomic Science, 1969, 17, 335-336.

GIBSON, A. R., FILBEY, R., \& GAZZANIGA, M. S. Hemispheric differences as reflected by reaction time. Federation Proceedings, 1970, 29, 658. (Abstract)

KLATZKY, R. L., \& ATKINSON, R. C. Memory scans based on alternative test stimulus representations. Perception \& Psychophysics, $1970,8,113-117$.

KLATZKY, R. L., JUOLA, J. F., \& ATKINSON, R. C. Test stimulus representation and experimental context effects in memory scanning. Institute for Mathematical Studies in the Social Sciences, Stanford University, Technical Report No. 160, 1970.

LEVY, J. Possible basis for the evolution of lateral specialization of the human brain. Nature, 1969, 224, 614-615.

LEVY-AGRESTI, J., \& SPERRY, R. W. Differential perceptual capacities in major and minor hemispheres. Proceedings of the National Academy of Sciences, 1968, 61. 1151.

MOSCOVITCH, M., \& CATLIN, J. Interhemispheric transmission of information: Measurement in normal man. Psychonomic Science, 1970, 18, 211-212.

STERNBERG, $S$. High-speed scanning in human memory. Science, 1966, 153, 652-654.

\title{
An investigation into changes in decision criteria and other details of a decision-making task
}

\author{
M. HAMMERTON \\ Applied Psychology Unit, Cambridge, England
}

A method is described for constructing a decision-making task which satisfies the formal assumptions of signal-detection theory. Two experiments using this task are described. It is shown that Ss did not maintain a constant likelihood-ratio criterion and, hence, that the subjective $d^{\prime}$ was always less than the true value, even though Ss knew what that value was. Introducing confidence judgments did not significantly affect $d^{\prime}$. It is suggested that the method described is a useful one for examining decision responses.

The purpose of this study was to construct a decision-making task whose characteristics matched the assumptions of signal detection theory (e.g. Tanner \& Swets, 1954) with the modification that the individual presentations of evidence to $S$ of "signal" or of "signal + noise" were plain and manifest. To this end, each presentation consisted of a two-digit number drawn from a known population of numbers. In each experiment, two such populations were used, both being Gaussian; and each presentation was drawn with equal probability from either. The population regarded as "noise" had a mean of 40: the "signal" population had a mean of 43.47 . and 50 in the several experiments performed so far; and all populations had a variance of 10 .

Each number was clearly displayed to the $\mathrm{S}$, the digits being self-luminous, $2.5 \mathrm{~cm}$ high and approximately $60 \mathrm{~cm}$ from $S$ 's eyes.

It will be noted that this experimental method satisfies the formal assumptions of signal detection theory; but that such necessary uncertainties as, e.g., the exact form of the distributions, are removed; and S's evidence is precisely determined. It is

Table 1

Group Median Values of Subjective $d^{\prime}$ and $\beta$

\begin{tabular}{llll}
\hline True d' & 0.95 & 2.21 & 3.16 \\
Subjective d & 0.69 & 1.64 & 2.90 \\
$\beta$ & 1.01 & 1.10 & 0.89 \\
\hline
\end{tabular}




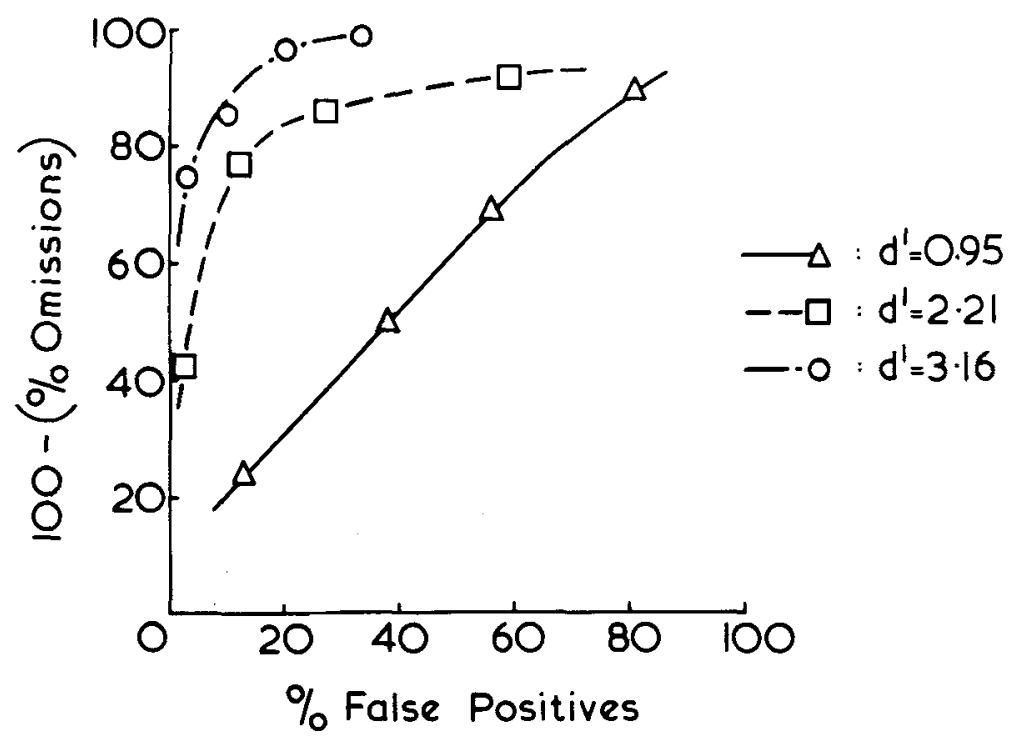

Fig. 1 Group median ROC curves for 3 values of d' care was taken to ensure that they did thus possible to examine in detail S's choices in a variety of precisely controlled conditions. Two experiments have so far been carried out. The first was specifically aimed at studying S's likelihood criteria; the second at the details of their ROC curves.

\section{METHOD \\ Experiment 1}

Ss were shown the apparatus, and it was explained to then that they would be shown numbers drawn randomly from two batches of numbers whose means they were told, i.e, the objective value of $d^{\prime}$ was given. They were asked, when each number appeared, to say "yes" if they thought it came from the group with the larger mean (i.e., was a "signal") and "no" if they thought the number came from the other group. Some Ss seemed to have difficulty understanding these instructions, and great understand before starting to test. Ss were always given knowledge of results, and the task was paced by them. Three groups of six Ss each took part in this experiment. For one group objective $\mathrm{d}^{\prime}$ was 0.95 , for another 2.21, and for the third 3.16. Responses to the last 100 numbers were scored in all cases.

Experiment 2

This differed from Experiment 1 in that

Ss were asked to give a confidence estimate instead of a simple yes-no response. The confidence rating was on a 5-point scale. with 1 being "surely a signal," 2 "probably a signal," 3 "don't know," 4 "probably noise," and 5 "surely noise." This code was written large on the wall beside the S. whose response was merely a number from 1 to 5 . Once again, three groups of six Ss were tested, the several groups having the same d's as those in Experiment 1. The task was again self-paced with knowledge of results.

\section{RESULTS \\ Experiment 1}

Each S's subjective d' was computed by adding the values of normal deviates corresponding to his proportion of misses and false positives (Welford, 1968). Similarly, each S's $\beta$ was computed as the ratio of the ordinates corresponding to his proportion of misses and false positives (Welford, 1968). Table 1 gives the median values of these quantities for each group, i.e., for each given $d^{\prime}$.

The sign test shows that all subjective d's are significantly less than the true value $(p<.05)$, while the $\beta$ s do not differ significantly from 1.

Examining the data in detail showed clearly why subjective $d^{\prime}$ was lower than true $\mathrm{d}^{\prime}$ : Ss do not maintain a constant

Table 2

Median Receiver Operating $d^{\prime}$ and $\beta$ s for Three Values of True $d^{\prime}$

Confidence Rating

\begin{tabular}{|c|c|c|c|c|c|c|c|c|}
\hline \multirow{2}{*}{$\begin{array}{c}\text { True } \\
\mathrm{d}^{\prime}\end{array}$} & \multicolumn{2}{|c|}{1} & \multicolumn{2}{|c|}{2} & \multicolumn{2}{|c|}{3} & \multicolumn{2}{|c|}{4} \\
\hline & $S \mathrm{~d}^{\prime}$ & $\beta$ & $S d^{\prime}$ & $\beta$ & $\mathrm{S} \mathrm{d}^{\prime}$ & $\beta$ & $S \mathrm{~d}^{\prime}$ & $\beta$ \\
\hline 0.95 & 0.45 & 1.38 & 0.52 & 1.05 & 0.58 & 0.91 & 0.44 & 0.67 \\
\hline 2.21 & 1.63 & 2.67 & 1.87 & 1.65 & 1.87 & 0.96 & 1.65 & 0.57 \\
\hline 3.16 & 2.44 & 3.00 & 2.32 & 1.24 & 2.59 & 0.41 & 2.82 & 0.21 \\
\hline
\end{tabular}

likelihood-ratio criterion. All but 1 of the 18 Ss varied their probability of responding "yes" witl the value of the "stimulus" presented. and only 2 did so monotonically. There was no sign of a "perfect Bayesian man."

\section{Experiment ?}

For each $S$ the percent of false positives and omissions corresponding to the first four confidence ratings were found. The medians of these values for the three groups, i.e.. for the three given values of $d^{\prime}$, are plotted in Fig. 1.

Table 2 gives the group median values of subjective $d^{\prime}$ and $\beta$ corresponding to the first four points of the three ROC curves, obtained as for Table 1 above.

It will be noted that the data of Table 2 generally follow the pattern of constant $d^{\prime}$ and decreasing $\beta$ as the confidence level changes, as predicted by theory. Likewise. the general form of the ROC curves is as predicted and frequently confirmed (e.g., Massaro, 1969). However, detailed examination of the several $S$ 's responses indicates that this is only statistically so. Some Ss exhibited violent changes of $\mathrm{d}^{\prime}$, one $\mathrm{S}$ maintained a constant $\beta$, and one refused to give any responses but " 1 " and "5."

It is important to note that $\mathrm{U}$ tests do not show the subjective $d^{\prime}$ s of Table 2 to differ significantly from those of Table 1. Thus there is no support for the contention that demanding judgments of confidence levels distorts the value of $d^{\prime}$. However, in all medians and in every individual $S$ but one, the subjective $d^{\prime}$ was less than the true value, even though all $S$ s knew what that value was. This seems to confirm that the variation in criterion remarked above constitutes a source of error not allowed for in signal-detection theory.

It should not be overlooked that it is a frequenct characteristic of "guessing" and "odds estimation" experiments that Ss consistently score below optimum. This is often so even when, as here, full information is presented to them (e.g., Dale, 1968).

It is evident that the present method can be applied to a number of situations, and a number of further investigations are in hand.

\section{REFERENCES}

DALE, H. C. A. Weighing evidence: An attempt to assess the efficiency of the human operator. Ergonomics, 1968, 11, 215-230.

MASSARO, $D$. W. The role of the decision system in sensory and memory experiments using confidence judgments. Perception \& Psychophysics, 1969, 5, 270-272.

TANNER, W. P., \& SWETS, J. A. A decision-making theory of visual detection. Psychological Review, 1954, 61, 401-409.

WELFORD, A. T. Fundamentals of skill. London: Methuen, 1968. 